\title{
Prenatal maternal antidepressants, anxiety, and depression and offspring DNA methylation: epigenome-wide associations at birth and persistence into early childhood
}

Andres Cardenas ${ }^{1 *+} \mathbb{D}$, Sabrina Faleschini ${ }^{2+}$, Andrea Cortes Hidalgo ${ }^{3}$, Sheryl L. Rifas-Shiman ${ }^{4}$, Andrea A. Baccarelli ${ }^{5}$, Dawn L. DeMeo ${ }^{6}$, Augusto A. Litonjua ${ }^{7}$, Alexander Neumann³, Janine F. Felix ${ }^{8,9,10}$, Vincent W. V. Jaddoe ${ }^{8,9,10,}$ Hanan El Marroun ${ }^{3,8}$, Henning Tiemeier ${ }^{3,9}$, Emily Oken ${ }^{1}$, Marie-France Hivert ${ }^{4,11}$ and Heather H. Burris ${ }^{12}$

\begin{abstract}
Background: Maternal mood disorders and their treatment during pregnancy may have effects on the offspring epigenome. We aim to evaluate associations of maternal prenatal antidepressant use, anxiety, and depression with cord blood DNA methylation across the genome at birth and test for persistence of associations in early and midchildhood blood DNA.
\end{abstract}

Methods: A discovery phase was conducted in Project Viva, a prospective pre-birth cohort study with external replication in an independent cohort, the Generation R Study. In Project Viva, pregnant women were recruited between 1999 and 2002 in Eastern Massachusetts, USA. In the Generation R Study, pregnant women were recruited between 2002 and 2006 in Rotterdam, the Netherlands. In Project Viva, 479 infants had data on maternal antidepressant use, anxiety, depression, and cord blood DNA methylation, 120 children had DNA methylation measured in early childhood ( 3 years), and 460 in mid-childhood ( 7 years). In the Generation R Study, 999 infants had data on maternal antidepressants and cord blood DNA methylation. The prenatal antidepressant prescription was obtained from medical records. At-mid pregnancy, symptoms of anxiety and depression were assessed with the Pregnancy-Related Anxiety Scale and the Edinburgh Postnatal Depression Scale in Project Viva and with the Brief Symptom Inventory in the Generation R Study. Genomewide DNA methylation was measured using the Infinium HumanMethylation450 BeadChip in both cohorts.

Results: In Project Viva, 2.9\% (14/479) pregnant women were prescribed antidepressants, 9.0\% (40/445) experienced high pregnancy-related anxiety, and 8.2\% (33/402) reported symptoms consistent with depression. Newborns exposed to antidepressants in pregnancy had $7.2 \%$ lower DNA methylation $\left(95 \% \mathrm{Cl},-10.4,-4.1 ; P=1.03 \times 10^{-8}\right)$ at $\mathrm{cg} 22159528$ located in the gene body of ZNF575, and this association replicated in the Generation R Study $(\beta=-2.5 \% ; 95 \% \mathrm{Cl}-4.2$, $-0.7 ; P=0.006)$. In Project Viva, the association persisted in early $(\beta=-6.2 \% ; 95 \% \mathrm{Cl}-10.7,-1.6)$ but not midchildhood. We observed cohort-specific associations for maternal anxiety and depression in Project Viva that did not replicate.

(Continued on next page)

\footnotetext{
* Correspondence: andres.cardenas@berkeley.edu

${ }^{+}$Andres Cardenas and Sabrina Faleschini contributed equally to this work.

${ }^{1}$ Division of Environmental Health Sciences, School of Public Health,

University of California, Berkeley, Berkeley, CA, USA

Full list of author information is available at the end of the article
}

(c) The Author(s). 2019 Open Access This article is distributed under the terms of the Creative Commons Attribution 4.0 International License (http://creativecommons.org/licenses/by/4.0/), which permits unrestricted use, distribution, and reproduction in any medium, provided you give appropriate credit to the original author(s) and the source, provide a link to the Creative Commons license, and indicate if changes were made. The Creative Commons Public Domain Dedication waiver (http://creativecommons.org/publicdomain/zero/1.0/) applies to the data made available in this article, unless otherwise stated. 
(Continued from previous page)

Conclusions: The ZNF575 gene is involved in transcriptional regulation but specific functions are largely unknown.

Given the widespread use of antidepressants in pregnancy, as well as the effects of exposure to anxiety and depression, implications of potential fetal epigenetic programming by these risk factors and their impacts on development merit further investigation.

Keywords: Maternal depression, Maternal anxiety, Antidepressants, DNA methylation, Fetal programming

\section{Background}

Anxiety and depression are common during pregnancy, affecting up to $8 \%$ and $12 \%$ of pregnant women, respectively [1-3]. Prenatal anxiety and depression are associated with poor perinatal outcomes including suboptimal fetal growth [4, 5] and preterm birth [6]. While generally thought to be safe, medications to treat mood disorders in pregnancy have been associated with risks of adverse long-term consequences for children including impaired neuromotor development [7] as well as behavioral and emotional problems [8-11].

Antidepressants such as selective serotonin reuptake inhibitors (SSRIs) are used to reduce symptoms of anxiety and depression in approximately $8 \%$ of US pregnant women [12]. Two recent reviews of the literature found that fetuses exposed to antidepressants such as SSRIs may have abnormal motor and heart rate activity during fetal development [13, 14]. It is well established that early-life environments may influence fetal and later child development [15]. Epigenetic processes during fetal development are one pathway by which environmental factors may affect phenotype later in life [16]. Whether antidepressants or the underlying psychopathology in pregnancy affects fetal programming through epigenetic processes such as DNA methylation remains unknown.

Epigenome-wide association studies (EWAS) can be a powerful tool to discover biomarkers of disease and to understand biologic processes [17]. Using an epigenomewide approach, we aimed to identify differences in DNA methylation in neonates associated with prenatal maternal antidepressant use, anxiety, and depression. We hypothesized that prenatal maternal exposure to antidepressants, anxiety, and depression would lead to differences in DNA methylation in cord blood that would persist into childhood.

\section{Results}

Overall, in Project Viva, 2.9\% (14/479) of women were prescribed antidepressants during pregnancy, 9.0\% (40/445) experienced high pregnancy-related anxiety, and $8.2 \%$ (33/402) reported symptoms consistent with depression in pregnancy. In the Generation R Study, there were 999 mother-infant pairs eligible for analyses, 1.4\% (14/999) were prescribed antidepressants, $5.8 \% \quad(56 / 969)$ experienced high anxiety, and 3.2\% (31/969) reported symptoms consistent with clinical depression in pregnancy. Demographic characteristics of participants in both cohorts are presented in Table 1.

In Project Viva, exposure to antidepressants was associated with DNA methylation differences at $130 \mathrm{CpG}$ sites that passed FDR $<0.05$, among which 16 sites also passed Bonferroni significance $\left(P<1.34 \times 10^{-7}\right)$ in models adjusted for maternal, parity, self-reported race, smoking during pregnancy, body mass index (BMI), mode of delivery, education and infant sex, gestational age at birth, and nucleated cell-type proportions (Table 2). In replication analyses in the Generation $\mathrm{R}$ Study, among Bonferroni significant sites discovered in Project Viva, we confirmed that DNA methylation of one of these sites, cg22159528, was significantly lower among infants whose mothers were prescribed antidepressants during pregnancy. This CpG site is located in the body of the Zinc Finger Protein 575 gene (ZNF575) on chromosome 19 and annotated to a $\mathrm{CpG}$ island. Specifically, in Project Viva, we observed that infants born to mothers prescribed antidepressant in pregnancy had 7.2\% lower DNA methylation $\left(95 \% \mathrm{CI}-10.4,-4.1 ; P=1 \times 10^{-8}\right)$ at this site and in the Generation R Study, exposed infants had $2.5 \%$ lower DNA methylation (95\% CI $-4.2,-0.7 ; P=0.006)$ at the same site in adjusted models. In the discovery cohort, we also observed an additional four CpG sites (cg01080902, cg04798919, cg10571104, and cg17970176) near cg22159528 in the ZNF575 gene that were nominally associated with antidepressant use $(P<0.05)$ in the replication cohort but did not reach a Bonferroni adjusted (0.05/ 16) levels of significance (Fig. 1). One other CpG site in the replication cohort (cg00367463; BEST4 gene) passed the $P<0.05$ criteria for replication but its effect estimate was in the opposite direction.

In Project Viva, we observed 13 individual CpG sites differentially methylated relative to high maternal pregnancy-related anxiety and three individual sites associated with prenatal maternal depression (FDR $<0.05$ ) but these associations were not robust to external replication in the Generation $\mathrm{R}$ Study (Additional file 1: Table S1). For single CpG analyses, the genomic inflation factor $(\lambda)$ was 0.87 for prenatal antidepressants, 1.17 for high anxiety, and 0.94 for depression indicating a reasonable fit (Additional file 2: Figure S1). As a secondary 
Table 1 Characteristics of the discovery cohort, Project Viva, and the independent replication cohort, Generation R Study

\begin{tabular}{|c|c|c|c|c|}
\hline \multirow{2}{*}{$\frac{\text { Characteristics }}{\text { Maternal }}$} & \multicolumn{2}{|c|}{ Discovery cohort Project Viva $N=479$} & \multicolumn{2}{|c|}{ Replication cohort Generation R Study N = 999} \\
\hline & \multicolumn{2}{|l|}{ Mean (SD) or $n(\%)$} & & \\
\hline Age, years & \multicolumn{2}{|l|}{$32.1(5.4)$} & \multicolumn{2}{|l|}{$32.2(4.3)$} \\
\hline Pre-pregnancy BMl, kg/m² & \multicolumn{2}{|l|}{$24.7(5.2)$} & \multicolumn{2}{|l|}{$23.4(3.9)$} \\
\hline Antidepressant use & \multicolumn{2}{|l|}{$14(2.9 \%)$} & \multicolumn{2}{|l|}{$14(1.4 \%)$} \\
\hline High anxiety & \multicolumn{2}{|l|}{$40(9.0 \%)^{\mathrm{a}}$} & \multicolumn{2}{|l|}{$56(5.8 \%)^{\mathrm{b}}$} \\
\hline Depression mid-pregnancy & \multicolumn{2}{|l|}{$33(8.2 \%)^{a}$} & \multicolumn{2}{|l|}{$31(3.2 \%)^{b}$} \\
\hline \multicolumn{5}{|l|}{ Race/ethnicity } \\
\hline & Non-Hispanic White & $341(71.2 \%)$ & Dutch & $931(93.2 \%)$ \\
\hline & Non-Hispanic Black & $56(11.7 \%)$ & Non-Dutch Western & $63(6.3 \%)$ \\
\hline & Hispanic & $37(7.7 \%)$ & Non-western & $5(0.5 \%)$ \\
\hline & Other & $45(9.4 \%)$ & - & \\
\hline College graduate or more education & \multicolumn{2}{|l|}{$317(66.2 \%)$} & \multicolumn{2}{|l|}{$669(67.0 \%)$} \\
\hline \multicolumn{5}{|l|}{ Smoking status } \\
\hline & Never & $327(68.3 \%)$ & Never during pregnancy & $772(77.3 \%)$ \\
\hline & Former & $100(20.9 \%)$ & Quit when pregnancy was known & $93(9.3 \%)$ \\
\hline & During pregnancy & $52(10.9 \%)$ & Continued during pregnancy & $134(13.4 \%)$ \\
\hline Perinatal/infant & \multicolumn{4}{|l|}{ Mean (SD) or $n(\%)$} \\
\hline Cesarean delivery & \multicolumn{2}{|l|}{$79(16.5 \%)$} & \multicolumn{2}{|l|}{$103(10.3 \%)$} \\
\hline Gestational age at delivery, weeks & \multicolumn{2}{|l|}{$39.8(1.4)$} & \multicolumn{2}{|l|}{$40.2(1.4)$} \\
\hline Birth weight-for-gestational age, $z$-score & \multicolumn{2}{|l|}{$0.27(1.0)$} & \multicolumn{2}{|l|}{$0.26(0.87)^{c}$} \\
\hline Female infant & \multicolumn{2}{|l|}{$229(47.8 \%)$} & \multicolumn{2}{|l|}{$484(48.4 \%)$} \\
\hline
\end{tabular}

${ }^{a} 34$ missing data on maternal anxiety and 77 missing data on maternal depression in Project Viva

b8 missing data on maternal depression and anxiety in the Generation R Study

c 1 missing data for birthweight-for-gestational age in Generation R Study

approach, we conducted regional analyses using DMRcate: we did not find any differentially methylated regions relative to prenatal antidepressant prescription, anxiety, or depression in the discovery cohort.

We evaluated the persistence of the observed association at cg22159528 in the ZNF575 gene for antidepressants and DNA methylation in Project Viva, in blood collected in early and mid-childhood. In adjusted models, prenatally exposed children $(n=4$ out of 120$)$ had $6.2 \%$ lower DNA methylation $\left(95 \% \mathrm{CI}-10.7\right.$ to $\left.-1.6 ; P=6.70 \times 10^{-3}\right)$ compared to non-exposed children in early childhood. This association was in the same direction but attenuated and non-significant in mid-childhood $(\beta=-3.7,95 \% \mathrm{CI}-8.8$ to $1.4 ; P=0.16)(n=12$ exposed out of 460$)$. Unadjusted differences in DNA methylation were similar to adjusted differences for exposed and unexposed infants at birth, early, and mid-childhood (Fig. 2).

To evaluate the potential neurological implications of our findings, we tested correlations between blood and brain DNA methylation using external reference data. DNA methylation at cg22159528 in the ZNF575 gene from over 70 adults showed positive correlations between blood and brain tissue of the prefrontal cortex $\left(r=0.54, P=6.45 \times 10^{-7}\right)$, entorhinal cortex $\left(r=0.41, \quad P=2.33 \times 10^{-4}\right)$, superior temporal gyrus $\left(r=0.49, P=7.87 \times 10^{-8}\right)$ but not the cerebellum ( $r=-0.01, P=0.97)$ (Fig. 3$)$. These results must be interpreted with caution given that reference blood and brain samples were collected from adults and might not accurately reflect variation in cord blood or early childhood blood samples with brain DNA methylation.

\section{Discussion}

Using an agnostic epigenome-wide approach, we observed differences in DNA methylation across multiple CpG sites for infants prenatally exposed to maternal antidepressants and replicated this observation at one CpG site. While $13 \mathrm{CpG}$ sites were associated with high maternal prenatal anxiety and three with prenatal maternal depression in Project Viva, we did not confirm these associations in the Generation R Study. In both Project Viva and Generation R Study, antidepressant prescription during pregnancy was associated with lower DNA methylation at a $\mathrm{CpG}$ site located within the ZNF575 gene body. Exposure to antidepressants during pregnancy was also associated with lower DNA methylation at this site in early childhood blood with a similar 
Table 2 Differentially methylated CpGs in umbilical cord blood DNA associated with prenatal maternal antidepressants in pregnancy

\begin{tabular}{|c|c|c|c|c|c|c|c|c|c|}
\hline \multirow[t]{2}{*}{ CpG } & \multirow[t]{2}{*}{ Chr } & \multirow[t]{2}{*}{$\begin{array}{l}\text { Genomic } \\
\text { Position }\end{array}$} & \multirow[t]{2}{*}{ Gene } & \multicolumn{2}{|c|}{ Discovery cohort Project Viva $(n=479)$} & \multirow[b]{2}{*}{$P$} & \multicolumn{3}{|c|}{$\begin{array}{l}\text { Replication cohort Generation R Study } \\
\left(n=999^{c}\right)\end{array}$} \\
\hline & & & & $\begin{array}{l}\text { Mean (SD) } \\
\text { \%-DNA } \\
\text { methylation }\end{array}$ & $\begin{array}{l}\text { Adjusted \% change in DNA } \\
\text { methylation }(95 \% \mathrm{Cl})^{\mathrm{a}}\end{array}$ & & $\begin{array}{l}\text { Mean (SD) } \\
\text { \%-DNA } \\
\text { methylation }\end{array}$ & $\begin{array}{l}\text { Adjusted \% change in } \\
\text { DNA methylation } \\
(95 \% \text { CI) }\end{array}$ & $P$ \\
\hline cg00367463 & 1 & $45,249,899$ & BEST4 & $1.9(0.4)$ & $0.26(0.16,0.37)$ & $2.01 \times 10^{-8}$ & $10.3(2.7)$ & $-0.53(-1.02,-0.03)$ & 0.04 \\
\hline $\operatorname{cg} 27566858$ & 1 & $208,084,099$ & CD34 & $2.0(0.3)$ & $0.27(0.15,0.39)$ & $3.13 \times 10^{-9}$ & $12.6(3.3)$ & $0.26(-0.53,1.06)$ & 0.52 \\
\hline cg03536711 & 1 & $221,509,067$ & LOC400804 & $53.3(11.4)$ & $-11.63(-15.80,-7.45)$ & $3.24 \times 10^{-8}$ & $54.9(9.3)$ & $2.25(-1.53,6.02)$ & 0.24 \\
\hline cg07729367 & 3 & $128,479,008$ & $R A B 7 A$ & $98.2(0.5)$ & $-0.49(-0.70,-0.27)$ & $1.08 \times 10^{-7}$ & $90.6(2.1)$ & $-0.31(-1.36,0.75)$ & 0.57 \\
\hline cg22065513 & 3 & $144,241,532$ & & $97.1(1.4)$ & $-1.22(-1.73,-0.72)$ & $4.11 \times 10^{-9}$ & $90.7(2.0)$ & $-0.36(-1.33,0.61)$ & 0.47 \\
\hline cg27299660 & 3 & $171,527,797$ & PLD1 & $1.7(0.4)$ & $0.77(0.49,1.04)$ & $1.19 \times 10^{-7}$ & $8.3(2.3)$ & $0.11(-0.55,0.77)$ & 0.75 \\
\hline cg14499053 & 7 & $19,158,954$ & & $2.2(0.5)$ & $-0.33(-0.45,-0.22)$ & $5.44 \times 10^{-8}$ & $11.5(2.1)$ & $0.35(-0.48,1.19)$ & 0.41 \\
\hline cg15881597 & 7 & $73,085,754$ & VPS37D & $40.1(4.9)$ & $-3.64(-5.09,-2.19)$ & $5.66 \times 10^{-9}$ & $47.3(4.7)$ & $-1.82(-4.06,0.42)$ & 0.11 \\
\hline cg06645921 & 12 & $8,025,394$ & SLC2A14 & $6.6(3.7)$ & $-2.38(-2.95,-1.81)$ & $9.11 \times 10^{-16}$ & NA & $N A$ & NA \\
\hline cg27161197 & 12 & $47,224,649$ & & $71.5(5.9)$ & $-5.17(-7.12,-3.23)$ & $1.77 \times 10^{-9}$ & $68.9(5.5)$ & $-2.01(-5.23,1.21)$ & 0.22 \\
\hline $\operatorname{cg} 25121621$ & 15 & $45,926,780$ & SQRDL & $19.6(2.9)$ & $2.74(1.82,3.67)$ & $2.71 \times 10^{-12}$ & $28.4(4.2)$ & $-0.80(-4.37,2.78)$ & 0.66 \\
\hline cg06358612 & 17 & $28,619,293$ & BLMH & $1.4(0.3)$ & $0.22(0.14,0.31)$ & $6.07 \times 10^{-8}$ & $8.1(1.4)$ & $-0.18(-0.71,0.34)$ & 0.49 \\
\hline cg22159528 & 19 & $44,039,727$ & ZNF575 & $51.0(6.8)$ & $-7.23(-10.36,-4.10)$ & $1.03 \times 10^{-8}$ & $54.7(5.9)$ & $-2.46(-4.23,-0.69)$ & 0.006 \\
\hline $\operatorname{cg} 12489353$ & 19 & $48,231,499$ & EHD2 & $79.3(14.5)$ & $-10(-15.7,-4.3)$ & $7.88 \times 10^{-8}$ & $77.2(10)$ & $-0.80(-6.49,4.88)$ & 0.78 \\
\hline cg11449935 & 20 & $35,202,477$ & TGIF2 & $2.2(0.4)$ & $0.29(0.16,0.43)$ & $4.20 \times 10^{-8}$ & $10.2(2.2)$ & $0.31(-0.52,1.13)$ & 0.47 \\
\hline cg18036763 & 22 & $45,404,910$ & PHF21B & $10.2(3.6)$ & $3.24(1.75,4.74)$ & $7.62 \times 10^{-8}$ & $6.2(1.7)$ & $0.38(-0.34,1.11)$ & 0.30 \\
\hline
\end{tabular}

Abbreviations: SD standard deviation, $C$ confidence interval, $N A$ excluded from the replication cohort (Generation $\mathrm{R}$ Study) after standard quality control

${ }^{a}$ Adjusted for maternal age, parity, race/ethnicity, smoking (never, former and during pregnancy), pre-pregnancy BMl, mode of delivery, education and infant sex, gestational age, and estimated cord blood nucleated cells (CD8, CD4, Mono, NK, B cells, granulocytes and nRBCs)

${ }^{\mathrm{b}}$ Adjusted for the same covariates as above and included sample plate as an additional covariate

'Refers to sample size for cg22159528, whereas other models from Generation R Study excluded samples that failed at specific CpG sites (smallest sample size $n=970$ for $\mathrm{cg} 18036763)$

magnitude of the effect. Using a blood and brain DNA methylation reference database, we observed moderate correlations among three brain regions with blood cells at the discovered and validated CpG site in the ZNF575 gene.

The Zinc Finger Protein 575 gene (ZNF575) is part of a large family of zinc finger proteins with multiple diverse functions that are abundant across multiple eukaryotic genomes [18]. This protein is involved in transcriptional regulation and has been previously associated with lung cancer [19]. Otherwise, there is very little known about the function of the ZNF575 gene and its role in health or development. This top finding was persistent in early, at approximately 3 years of age, but not in mid-childhood in the discovery cohort. This is important, as the first 1000 days of life represent a period of rapid development and vulnerability able to influence the life course further stressing the need to fully characterize the function of the ZNF575 gene.

There were an additional $15 \mathrm{CpG}$ sites in cord blood associated with maternal antidepressant use that survived Bonferroni correction in Project Viva, but they were not replicated in The Generation R Study. Six prior studies of in utero antidepressant exposure and offspring DNA methylation were recently systematically reviewed by Viuff et al. [20]. The authors concluded that there was no consistent association among studies and highlighted the need for untargeted epigenetic assays with external validation [20]. None of the prior studies reported differentially methylated sites at/near ZNF575. Three of the studies used a candidate gene approach [21-23]; two used an earlier epigenome-wide array which analyzed only 27,000 CpG sites [24, 25]. Only one prior study by Non et al. examined associations between maternal SSRI use and offspring DNA methylation using the same DNA methylation platform as we did [26]. They used a case-control design of 22 exposed infants and 23 unexposed infants and found no significant association between SSRIs and offspring DNA methylation. In addition, Non and colleagues selected infants exposed to SSRIs that differ from the medications used in our population and did not adjust for cell-type composition. Lack of consistency found in the literature may be a result of differences in study design, population, technology for DNA methylation assessment, and smaller sample sizes as well as exposure timing and ascertainment.

A few of the cohort-specific associations of high levels of pregnancy-related anxiety with DNA methylation in Project Viva were consistent with prior literature. For 


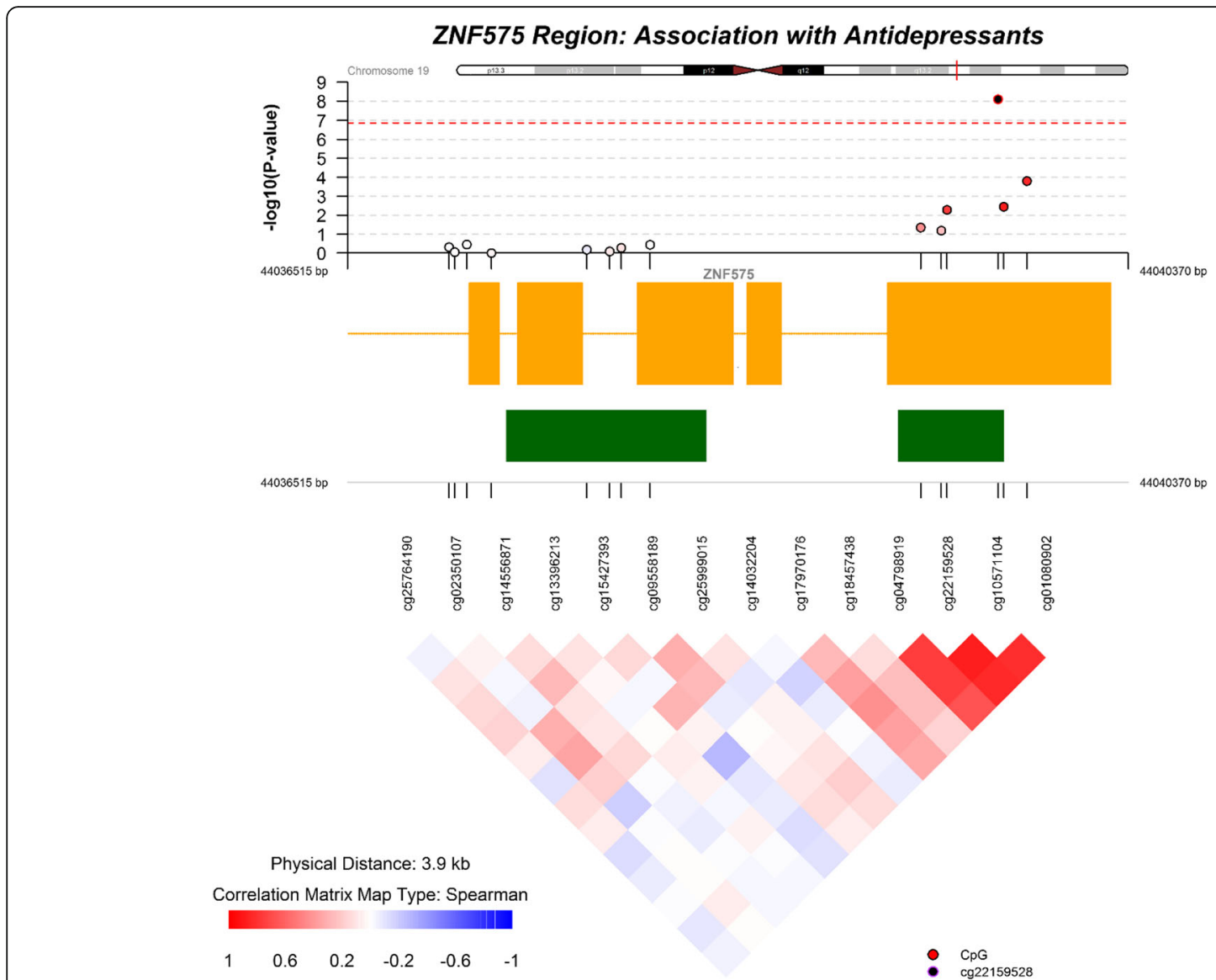

Fig. 1 Regional Manhattan plot for the adjusted association of prenatal maternal antidepressants and umbilical cord blood DNA methylation within ZNF575 gene region in Project Viva (orange squares indicate exons; orange lines indicate introns; green squares indicate CpG islands)
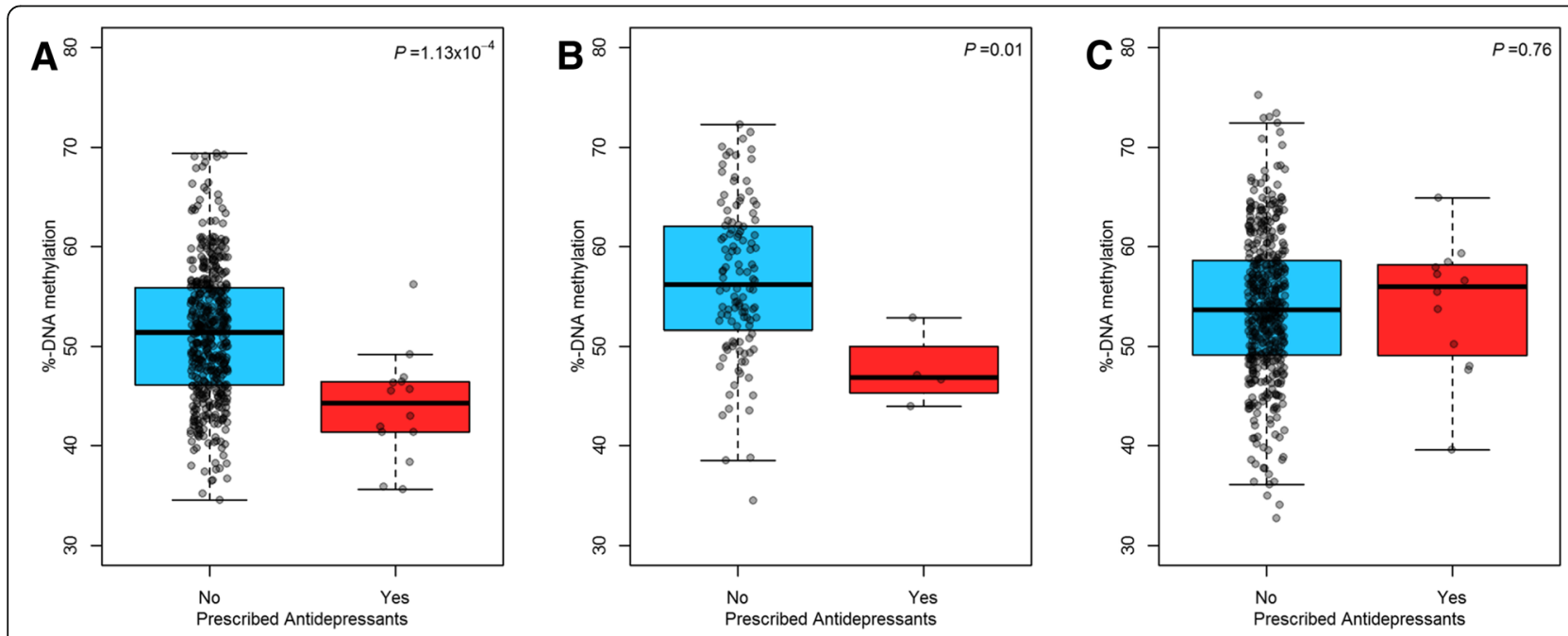

Fig. 2 Unadjusted \%-DNA methylation distribution for antidepressants exposed and unexposed infants at the replicated CpG site (cg22159528) in the ZNF575 gene and unadjusted Wilcoxon-rank sum test $P$ value in the discovery cohort, Project Viva, measured at three time points: a umbilical cord blood $(n=479)$, b early childhood $(n=120)$, and $\mathbf{c}$ mid-childhood peripheral blood $(n=460)$. One hundred twelve participants in early childhood also had cord blood measurements, and 235 participants from mid-childhood also had cord blood measurements 

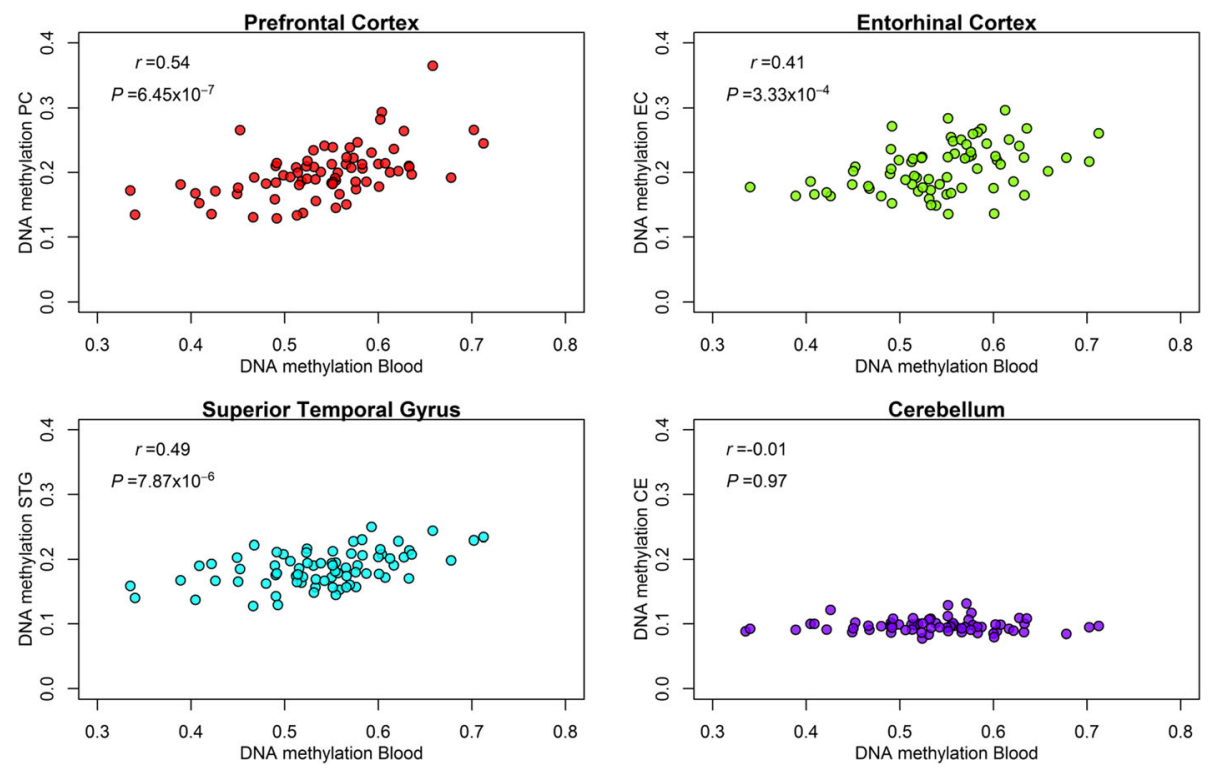

Fig. 3 Scatterplots and correlations for cg22159528 (ZNF575 gene) methylation levels of blood DNA and four brain regions: prefrontal cortex (PC, $n=74)$, entorhinal cortex (EC, $n=71)$, superior temporal gyrus (STG, $n=75)$, and cerebellum (CE, $n=71)$. Samples from external paired dataset of blood and brain tissue of adults [51].

example, a CpG site in the glial cell-derived neurotrophic factor (GDNF) gene showed higher DNA methylation compared to low or moderate pregnancy-related anxiety. Using blood samples, a study on inflammatory markers of women with antenatal depression found DNA methylation at another CpG site near GDNF to be higher among depressed pregnant women [27]. Also, in a mouse model, DNA methylation of the GDNF gene in experimentally stressed mice has been shown to be differentially methylated relative to stress. These experiments also showed that chronic stress reduced levels of a histone modification, $\mathrm{H} 3 \mathrm{~K} 4 \mathrm{me} 3$, in the promoter region of the GDNF gene and this effect was reversed by antidepressants [28].

Our study has several limitations. In Project Viva, over seven types of antidepressants were used during pregnancy with some women prescribed more than one single type, although 12/14 were SSRIs (Additional file 1: Table S2). In the Generation R Study, antidepressant prescriptions were limited to tricyclic antidepressants and SSRIs. In addition, in the Generation R Study, general anxiety during pregnancy was ascertained while pregnancy-related anxiety was evaluated in Project Viva. These are different scales and could capture different sources and levels of anxiety. Moreover, we measured DNA methylation only in blood and it is likely that blood may not accurately reflect DNA methylation variability in other relevant tissues. However, we used external brain and blood reference DNA methylation data to compare correlations at the externally replicated site. Our hypothesis was based on DNA methylation programming during fetal development. However, another possibility is that of cellular polycreodism-or the systematic variability of cell fate to yield a distinctive repertoire of cells [29]. Yet, without experimental data, it is impossible to determine the true causal effects of these exposures in the epigenome and therefore results should be interpreted as biomarkers. It would be nearly impossible to conduct a randomized trial for these prenatal maternal exposures and conditions. Additionally, timing, severity, and accuracy of self-reported depression and pregnancy-related anxiety along with medication adherence for antidepressants and repeated exposure could introduce substantial exposure misclassification making it challenging to capture underlying associations. Further, any study of the effects of medications can be affected by confounding by indication. Specifically, it may be the most depressed or anxious women who were treated with antidepressants and that those underlying causes were truly responsible for the observed associations. This issue is further complicated by the small overlap of women who exhibited symptoms of anxiety $(n=2)$ or depression $(n=4)$ among the treated women in Project Viva. The two cohorts also differed from one another, especially with respect to ethnicity (Table 1), which may have limited our ability to replicate findings across different populations. Lastly, our antidepressantexposed sample was small, limiting statistical power.

Our study also has important strengths. First, we implemented an epigenome-wide approach to agnostically capture associations with a relatively large sample size. Our prospective design reduces the chance of bias 
that might arise from case-control studies and allowed us to collect valuable confounder information early during pregnancy. Another major strength is the replication of findings in an independent birth cohort. Our use of an external reference dataset demonstrated moderate to strong correlations between DNA methylation of blood and three brain regions in the replicated site (ZNF575), suggesting that this finding may be relevant to long-term mental health or neurodevelopment. Yet, more work is needed to fully characterize the function of the ZNF575 gene.

\section{Conclusion}

In conclusion, we found DNA methylation of the ZNF575 gene in infant cord blood to be associated with maternal antidepressant use in pregnancy in two independent cohorts. We also demonstrated that this association persists into early childhood. These findings warrant further study to confirm the association and to determine its clinical significance.

\section{Methods}

\section{Discovery cohort: Project Viva}

We studied mother-child pairs participating in Project Viva, a prospective pre-birth cohort study recruited between 1999 and 2002 from Atrius Harvard Vanguard Medical Associates in MA, USA [30]. Mothers provided written, informed consent, and the institutional review board of Harvard Pilgrim Health Care approved the study. Of the total 2128 singleton births, there were 485 infants with cord blood DNA methylation data and information on prenatal maternal antidepressants, anxiety, and depression. We excluded 6 infants with gestational age $<34$ weeks and analyzed 479 mother-infant pairs with cord blood DNA methylation. We evaluated persistence of epigenetic associations observed at birth in 120 children ( $n=112$ included in cord blood analyses) with peripheral blood DNA methylation measurements from early childhood (mean 3.4 years, range 2.9 to 5.3) and 460 children $(n=235$ included in cord blood analyses) with peripheral blood DNA samples from midchildhood (mean 7.9 years, range 6.7 to 10.5).

We defined women as exposed to antidepressants if the medical record included a prescription during pregnancy (Additional file 1: Table S2). To assess anxiety, at the mid-pregnancy visit, we administered the 7-item Pregnancy-Related Anxiety Scale (PRAS) [31]. Answers are on a 4-point Likert scale (very much, moderately, somewhat, and not at all). The scale captures worry about fetal growth, health, and delivery method. The PRAS specifies three categories of anxiety levels (low, moderate, and high) with good reliability (Cronbach alpha $=0.78)$ [32]. We classified mothers as having high pregnancy-related anxiety if they chose "very much" to three or more questions on the PRAS and all other women served as the reference group. To assess depression at the mid-pregnancy visit, we administered the Edinburgh Postnatal Depression Scale (EPDS) [33], a 10-item questionnaire screening for depressive symptoms. Answers are on a 4-point Likert scale from 0 to 3. The EPDS is a validated screener for probable depression but it is not intended to diagnose clinical depression. The scale has been validated in pregnant women and has a sensitivity of $86 \%$ and a specificity of $78 \%$ for the diagnosis of depression [33, 34]. A score $\geq 13$ on the $0-30$ scale indicates probable prenatal depression $[35,36]$.

To assess DNA methylation, we used umbilical cord blood collected at delivery and whole blood samples from early and mid-childhood visits. Technicians extracted DNA using the Qiagen Puregene Kit (Valencia, CA) and stored aliquots at $-80^{\circ} \mathrm{C}$ until analysis. DNA underwent sodium bisulfite conversion using the EZ DNA Methylation-Gold Kit (Zymo Research, Irvine, CA). Samples were shipped to Illumina Inc. and analyzed for DNA methylation at $>485,000$ CpG sites simultaneously using the Infinium Human Methylation450 BeadChip (Illumina, San Diego, CA).

We used a two-stage algorithm in which we randomized 12 samples to each chip and then randomly assigned eight chips to each of the 15 plates used to ensure balance by sex across chips and plates. We excluded samples as potentially mislabeled if they were mismatched on sex, genotype or were deemed to be low in quality. Background correction and dye-bias equalization was performed via the normal-exponential out-of-band (noob) method [37], and a $\beta$-mixture quantile intra sample normalization procedure $(B M I Q)$ was applied to the data to reduce the potential bias that can arise from probe design [38]. For each CpG site, methylation is reported as average $\beta$ value $=M /(M+U+\varepsilon)$, where $M$ and $U$ represent the average fluorescence intensity from each probe corresponding to the methylated and unmethylated target CpG and $\varepsilon=100$, a small quantity to protect against division by zero. Thus, the average $\beta$ value is an interval-scaled quantity between zero and one interpreted as the fraction of DNA molecules whose target $\mathrm{CpG}$ is methylated across all nucleated cells. We excluded individual probes if they had non-significant detection $P$ values $(P>0.05)$ for more than $1 \%$ of the samples. Additionally, non-CpG probes (probes for SNPs (rs) and methylated sites other than cytosine (ch)), probes in $\mathrm{X}$ and $\mathrm{Y}$ chromosomes, SNP-associated probes at either the single base extension or within the target region were removed for SNPs that have a minor-allele frequency of $>5 \%$. Any probe with a SNP $\leq 10$ base pairs was excluded using annotation from the Bioconductor package IlluminaHumanMethylation450kanno.ilmn12.hg19 that utilized information from dbSNP. Previously identified 
non-specific and cross-reactive probes within the array along with polymorphic CpG sites were also excluded from the analysis [39]. We excluded individual probes with values greater than three times the interquartile range (IQR) from the 75th percentile or values less than three times the IQR from the 25th percentile to eliminate potential DNA methylation outliers. We used ComBat [40] to correct for technical variability from plate and scanner. We visually inspected the effectiveness of adjustment for batch using principal components before and after batch adjustment. We calculated the genomic inflation factor $(\lambda)$ for all three EWAS to evaluate systemic biases.

After quality control, there were 372,563 loci for analysis. We logit-transformed methylation values on the $\beta$ values (bounded between 0 and 100\%) to $M$ values prior to analyses as previously described to be more appropriate for the differential analysis of DNA methylation [41] but report results as \%-change in DNA methylation for interpretability.

\section{Replication cohort: the Generation R Study}

We pursued external replication of the top differentially methylated sites in Project Viva in an independent birth cohort study, the Generation R Study, based in Rotterdam, the Netherlands. For the Generation R Study, all pregnant women living in Rotterdam with an expected delivery date between April 2002 and January 2006 were asked to participate. In total, 9778 mothers were enrolled [42]. Cord blood DNA methylation was measured using Illumina Infinium HumanMethylation450 BeadChip (Illumina Inc., San Diego, USA).

Preparation and normalization of the HumanMethylation450 BeadChip array data were performed according to the CPACOR workflow [43] using the software package $R$ [44]. In detail, the idat files were read using the minfi package. Probes that had a detection $P$ value above background (based on the sum of methylated and unmethylated intensity values) $\geq 1 \times 10^{-16}$ were set to missing per array. Next, the intensity values were stratified by autosomal and non-autosomal probes and quantile normalized for each of the six probe type categories separately: type II red/green, type I methylated red/ green, and type I unmethylated red/green. Beta values were calculated as the proportion of methylated intensity value on the sum of methylated + unmethylated +100 intensities. Arrays with observed technical problems such as failed bisulfite conversion, hybridization or extension, and arrays with a mismatch between sex of the proband and sex determined by the chr $\mathrm{X}$ and $\mathrm{Y}$ probe intensities were removed from subsequent analyses. Additionally, only arrays with a call rate $>95 \%$ per sample were processed further.

A subset of $N=999$ mother-child pairs had complete information on maternal antidepressant use in pregnancy, and $N=969$ had complete information on maternal depression and anxiety. Maternal prenatal depression and anxiety were assessed at 20 weeks of pregnancy with the Brief Symptom Inventory $[45,46]$. This questionnaire comprises 53 items which provided nine scales of various psychiatric symptoms. The scale has a global index and includes two subscales for anxiety and depressive symptoms. The subscales for anxiety and depression contained six items each on a 5 -point scale, from 0 to 4 where a higher score indicates a higher level of symptoms. Antidepressant use was reported during each trimester of pregnancy using a self-reported questionnaire. Use of SSRI was confirmed with prescription records from pharmacies with participant consent. These measurements have been previously described in detail $[42,47,48]$.

\section{Statistical analyses}

For each covariate in both discovery and replication cohorts, we calculated means and standard deviations (SD), or sample sizes and percentages, to describe the discovery and replication cohorts. In the discovery cohort, we performed epigenome-wide DNA methylation analyses on a CpG-by-CpG basis to assess DNA methylation differences at each site in cord blood relative to prenatal maternal exposure to (1) antidepressant prescription, (2) anxiety, and (3) depression compared to non-exposed infants. We used separate robust linear regression models with heteroskedasticity-consistent estimators to model the methylation levels of each individual $\mathrm{CpG}$ on the $M$ value scale as the dependent variable and antidepressants, high pregnancy-related anxiety, and depression as predictors. We adjusted all regression models for variables selected a priori: maternal age, parity, self-reported race, smoking during pregnancy, body mass index (BMI), mode of delivery, education and infant sex, gestational age at birth, and nucleated cell-type proportions in cord blood (CD8+ $\mathrm{T}$ cells, CD4+ T cells, monocytes, natural killer cells, B cells, granulocytes, and nucleated red blood cells for cord blood analyses) estimated from the DNAm data using minfi [49]. Statistical significance for the CpG-by-CpG analyses was adjusted by controlling the false discovery rate at $5 \%(\mathrm{FDR}<0.05)$ for each of the three-independent epigenome-wide analyses. As secondary analyses, we tested for differentially methylated regions in relationship to antidepressant prescription, anxiety, and depression using DMRcate [50] with an FDR $<0.05$.

Similarly, in the replication cohort, we fit a robust linear regression with each of the top CpGs from discovery as the outcome for each prenatal maternal exposure and adjusted for similar covariates as we had in discovery. We tested CpG sites associated with prenatal maternal antidepressant use that passed 
Bonferroni correction in the discovery cohort due to an early departure from the expected uniform distribution for this EWAS (Additional file 2: Figures S1-S2) and for depression and anxiety among significant differentially methylated sites that passed FDR $<0.05$. In replication analyses, we deemed a $P<0.05$ as statistically significant in addition to having the association be consistent in direction with the discovery cohort.

We also evaluated the persistence of associations in early and mid-childhood in Project Viva by carrying forward individual loci found to be associated with DNA methylation in cord blood analyses that also replicated in the Generation R Study. Persistence of DNA methylation differences was evaluated in peripheral blood samples collected during early and mid-childhood using multivariate robust linear regression models adjusting for the same covariates as cord blood models with the addition of child age at the time of the blood draw. We considered $P<0.05$ as statistically significant for the persistence of epigenetic alterations in early or mid-childhood peripheral blood analyses. We also investigated unadjusted DNA methylation differences between exposed and unexposed children using boxplots and a Wilcoxon-rank sum test. We present the unadjusted distribution of DNA methylation levels in boxplots by antidepressant prescription given the relative small number of exposed infants. All analyses were carried out using the $\mathrm{R}$ statistical package, version 3.4.1 (www.r-project.org/).

\section{Blood-brain DNA methylation samples}

We evaluated co-variation between blood DNA methylation and methylation levels of brain regions using publicly available data from the Gene Expression Omnibus (GEO) repository (GSE59685). Briefly, to generate reference data, investigators collected whole blood samples prior to death and matched those samples to postmortem samples from the prefrontal cortex, entorhinal cortex, superior temporal gyrus and cerebellum of $N=75$ men and women $(40-105$ years old) [51]. They measured DNA methylation using the Illumina HumanMethylation450 BeadChip Array. Scatter plots and person correlation coefficients for the relationship between blood and brain DNA methylation was examined among sites that replicated in the external cohort.

\section{Additional files}

Additional file 1: Table S1. Differentially methylated CpG sites in umbilical cord blood DNA associated with high anxiety and depression (FDR $<0.05$ for the discovery cohort, Project Viva) and replication results from the Generation R Study. Table S2. Type of prenatal maternal antidepressants prescribed to the 14 unique participants in Project Viva. (DOCX 19 kb)

Additional file 2: Figure S1. Quantile-Quantile plots of observed vs expected $P$ values and genomic inflation factor $(\lambda)$ for Epigenome-Wide Associations of prenatal maternal A) prenatal antidepressants B) high pregnancy-related anxiety and C) depression. Figure S2. Manhattan plots for Epigenome-Wide Associations of prenatal maternal A) antidepressants B) high pregnancy-related anxiety and C) depression. (DOCX $852 \mathrm{~kb}$ )

\section{Abbreviations}

BMI: Body mass index; Cl: Confidence interval; EPDS: Edinburgh Postnatal Depression Scale; EWAS: Epigenome-wide association study;

PRAS: Pregnancy-Related Anxiety Scale; SD: Standard deviation;

SSRIs: Serotonin reuptake inhibitors; ZNF575: Zinc Finger Protein 575 gene

\section{Acknowledgements}

We gratefully acknowledge the contribution of general practitioners, hospitals, midwives, and pharmacies in Rotterdam and the participants and staff of Project Viva. We thank Mr. Michael Verbiest, Ms. Mila Jhamai, Ms. Sarah Higgins, Mr. Marijn Verkerk and Dr. Lisette Stolk for their help in creating the EWAS database. We thank Dr. A. Teumer for his work on the quality control and normalization scripts. The generation and management of the Illumina $450 \mathrm{~K}$ methylation array data (EWAS data) for the Generation R Study was executed by the Human Genotyping Facility of the Genetic Laboratory of the Department of Internal Medicine, Erasmus MC, the Netherlands.

\section{Funding}

This work was supported by the US National Institutes of Health grants K23ES022242, R01 NR013945, R01 HL111108. Project Viva is additionally supported by UG3OD023286, R01 HD034568. The Generation R Study is conducted by the Erasmus Medical Center in close collaboration with the Erasmus University Rotterdam, Faculty of Social Sciences, the Municipal Health Service Rotterdam area, and the Stichting Trombosedienst and Artsenlaboratorium Rijnmond (STAR), Rotterdam. The general design of the Generation R Study is made possible by financial support from the Erasmus Medical Center, Rotterdam, the Erasmus University Rotterdam, the Netherlands Organization for Health Research and Development and the Ministry of Health, Welfare and Sport. The EWAS data was funded by a grant to WWJ from the Netherlands Genomics Initiative (NGI)/ Netherlands Organisation for Scientific Research (NWO) Netherlands Consortium for Healthy Aging (NCHA; project nr. 050-060-810), by funds from the Genetic Laboratory of the Department of Internal Medicine, Erasmus MC, and by a grant from the National Institute of Child and Human Development (R01HD068437). V.W.J. received a grant from the Netherlands Organization for Health Research and Development (VIDI 016.136.361) and a Consolidator Grant from the European Research Council (ERC-2014-CoG-648916). This project received funding from the European Union's Horizon 2020 research and innovation programme (733206, LifeCycle and 633595, DynaHEALTH). A. Neumann and H. Tiemeier are supported by a grant of the Dutch Ministry of Education, Culture, and Science and the Netherlands Organization for Scientific Research (NWO grant No. 024.001.003, Consortium on Individual Development). The work of H. Tiemeier is further supported by the European Union Seventh Framework Program (FP7/2007-2013): ACTION: Aggression in Children: Unravelling gene-environment interplay to inform Treatment and InterventiON strategies (grant number 602768).

\section{Availability of data and materials}

The datasets generated and analyzed during the current study are not publicly available because this study began in 1999 and we did not obtain consent for such public release of genetic/epigenetic data. Data are available from the corresponding author or Project Viva study team (project_viva@hphc.org) upon reasonable request.

\section{Authors' contributions}

$\mathrm{HHB}$ and $\mathrm{AC}$ conceived and designed the study. AC analyzed and interpreted the data regarding Project Viva and wrote the manuscript. SF wrote the manuscript and helped interpret results. $\mathrm{ACH}$ analyzed and interpreted the data regarding the Generation R Study. HHB, ACH, SLRS, AAB, DLD, AAL, AN, JFF, WWJ, HEM, HT, EO, and MFH supervised the project and reviewed the manuscript. All authors read and approved the final manuscript.

\section{Ethics approval and consent to participate}

Project Viva study protocol was approved by the institutional review board of Harvard Pilgrim Health Care. The Generation R study protocol was approved by the Medical Ethical Committee of the Erasmus Medical Centre, Rotterdam. Written informed consent was obtained for all participants. 


\section{Consent for publication}

Not applicable.

\section{Competing interests}

The authors declare that they have no competing interests.

\section{Publisher's Note}

Springer Nature remains neutral with regard to jurisdictional claims in published maps and institutional affiliations.

\section{Author details}

${ }^{1}$ Division of Environmental Health Sciences, School of Public Health, University of California, Berkeley, Berkeley, CA, USA. ${ }^{2}$ School of Psychology, Laval University, Quebec, QC, Canada. ${ }^{3}$ Department of Child and Adolescent Psychiatry and Psychology, Erasmus MC - Sophia Children's Hospital, Rotterdam, the Netherlands. ${ }^{4}$ Division of Chronic Disease Research Across the Lifecourse, Department of Population Medicine, Harvard Medical Schoo and Harvard Pilgrim Health Care Institute, Boston, MA, USA. ${ }^{5}$ Department of Environmental Health Sciences, Mailman School of Public Health, Columbia University, New York, NY, USA. ${ }^{6}$ Channing Division of Network Medicine, Department of Medicine, Brigham and Women's Hospital, Harvard Medical School, Boston, MA, USA. 7 Division of Pediatric Pulmonary Medicine, University of Rochester Medical Center, Rochester, NY, USA. ${ }^{8}$ Generation R Study Group, Erasmus MC, University Medical Center Rotterdam, Rotterdam, the Netherlands. ${ }^{9}$ Department of Epidemiology, Erasmus MC, University Medical Center Rotterdam, Rotterdam, the Netherlands. ${ }^{10}$ Department of Pediatrics, Erasmus MC, University Medical Center Rotterdam, Rotterdam, the Netherlands. " Diabetes Unit, Massachusetts General Hospital, Boston, MA, USA. ${ }^{12}$ Division of Neonatology, Department of Pediatrics, Children's Hospital of Philadelphia and Perelman School of Medicine, University of Pennsylvania, Philadelphia, PA, USA.

\section{Received: 1 August 2018 Accepted: 12 March 2019}

Published online: 29 March 2019

\section{References}

1. Andersson L, Sundström-Poromaa I, Bixo M, Wulff M, Bondestam K, Åström M. Point prevalence of psychiatric disorders during the second trimester of pregnancy: a population-based study. American Journal of Obstetrics \& Gynecology. 2003;189(1):148-54.

2. Bennett HA, Einarson A, Taddio A, Koren G, Einarson TR. Prevalence of depression during pregnancy: systematic review. Obstet Gynecol. 2004; 103(4):698-709.

3. Ross LE, McLean LM, Psych C. Anxiety disorders during pregnancy and the postpartum period: a systematic review. Depression. 2006;6(9):1-14.

4. Ciesielski TH, Marsit CJ, Williams SM. Maternal psychiatric disease and epigenetic evidence suggest a common biology for poor fetal growth. BMC Pregnancy and Childbirth. 2015;15(1):192.

5. Henrichs J, Schenk J, Roza S, Van den Berg M, Schmidt H, Steegers E, et al. Maternal psychological distress and fetal growth trajectories: the Generation R Study. Psychol Med. 2010;40(4):633-43.

6. Provenzi L, Guida E, Montirosso R. Preterm behavioral epigenetics: a systematic review. Neurosci Biobehav Rev. 2018;84:262-71.

7. van Batenburg-Eddes T, de Groot L, Huizink AC, Steegers EA, Hofman A, Jaddoe WW, et al. Maternal symptoms of anxiety during pregnancy affect infant neuromotor development: the generation $\mathrm{R}$ study. Dev Neuropsychol. 2009;34(4):476-93.

8. O'connor TG, Heron J, Golding J, Glover V. Maternal antenatal anxiety and behavioural/emotional problems in children: a test of a programming hypothesis. J Child Psychol Psychiatry. 2003;44(7):1025-36.

9. Van den Bergh BR, Van Calster B, Smits T, Van Huffel S, Lagae L. Antenatal maternal anxiety is related to HPA-axis dysregulation and self-reported depressive symptoms in adolescence: a prospective study on the fetal origins of depressed mood. Neuropsychopharmacology. 2008;33(3):536.

10. Velders FP, Dieleman G, Cents RA, Bakermans-Kranenburg MJ, Jaddoe VW, Hofman A, et al. Variation in the glucocorticoid receptor gene at rs41423247 moderates the effect of prenatal maternal psychological symptoms on child cortisol reactivity and behavior. Neuropsychopharmacology. 2012;37(11):2541.
11. Glover V. Maternal depression, anxiety and stress during pregnancy and child outcome; what needs to be done. Best Practice \& Research Clinical Obstetrics \& Gynaecology. 2014;28(1):25-35.

12. Andrade SE, Raebel MA, Brown J, Lane K, Livingston J, Boudreau D, et al. Use of antidepressant medications during pregnancy: a multisite study. American Journal of Obstetrics \& Gynecology. 2008;198(2):194.e1-5.

13. Glover ME, Clinton SM. Of rodents and humans: a comparative review of the neurobehavioral effects of early life SSRI exposure in preclinical and clinical research. Int J Dev Neurosci. 2016;51:50-72.

14. El Marroun H, White T, Verhulst FC, Tiemeier H. Maternal use of antidepressant or anxiolytic medication during pregnancy and childhood neurodevelopmental outcomes: a systematic review. European Child \& Adolescent Psychiatry. 2014;23(10):973-92.

15. Constantinof A, Moisiadis VG, Matthews SG. Programming of stress pathways: a transgenerational perspective. J Steroid Biochem Mol Biol. 2016; 160:175-80.

16. Burris HH, Baccarelli AA. Environmental epigenetics: from novelty to scientific discipline. J Appl Toxicol. 2014;34(2):113-6.

17. Greally JM, Drake AJ. The current state of epigenetic research in humans: promise and reality. JAMA Pediatr. 2017;171(2):103-4.

18. Laity $J H$, Lee BM, Wright PE. Zinc finger proteins: new insights into structural and functional diversity. Curr Opin Struct Biol. 2001;11(1):39-46.

19. Chang JS, Wrensch MR, Hansen HM, Sison JD, Aldrich MC, Quesenberry CP $\mathrm{Jr}$, et al. Base excision repair genes and risk of lung cancer among San Francisco Bay Area Latinos and African-Americans. Carcinogenesis. 2008; 30(1):78-87.

20. Viuff A-CF, Pedersen LH, Kyng K, Staunstrup NH, Børglum A, Henriksen TB. Antidepressant medication during pregnancy and epigenetic changes in umbilical cord blood: a systematic review. Clin Epigenetics. 2016;8(1):94.

21. Oberlander TF, Weinberg J, Papsdorf M, Grunau R, Misri S, Devlin AM. Prenatal exposure to maternal depression, neonatal methylation of human glucocorticoid receptor gene (NR3C1) and infant cortisol stress responses. Epigenetics. 2008;3(2):97-106.

22. Soubry A, Murphy SK, Huang Z, Murtha A, Schildkraut JM, Jirtle RL, et al. The effects of depression and use of antidepressive medicines during pregnancy on the methylation status of the IGF2 imprinted control regions in the offspring. Clin Epigenetics. 2011;3(1):2.

23. Devlin AM, Brain U, Austin J, Oberlander TF. Prenatal exposure to maternal depressed mood and the MTHFR C677T variant affect SLC6A4 methylation in infants at birth. PLoS One. 2010;5(8):e12201.

24. Schroeder JW, Smith AK, Brennan PA, Conneely KN, Kilaru V, Knight BT, et al. DNA methylation in neonates born to women receiving psychiatric care. Epigenetics. 2012;7(4):409-14.

25. Gurnot C, Martin-Subero I, Mah SM, Weikum W, Goodman SJ, Brain U, et al. Prenatal antidepressant exposure associated with CYP2E1 DNA methylation change in neonates. Epigenetics. 2015;10(5):361-72.

26. Non AL, Binder AM, Kubzansky LD, Michels KB. Genome-wide DNA methylation in neonates exposed to maternal depression, anxiety, or SSRI medication during pregnancy. Epigenetics. 2014;9(7):964-72.

27. Edvinsson Å, Bränn E, Hellgren C, Freyhult E, White R, Kamali-Moghaddam $M$, et al. Lower inflammatory markers in women with antenatal depression brings the M1/M2 balance into focus from a new direction. Psychoneuroendocrinology. 2017;80:15-25.

28. Uchida S, Hara K, Kobayashi A, Otsuki K, Yamagata H, Hobara T, et al. Epigenetic status of Gdnf in the ventral striatum determines susceptibility and adaptation to daily stressful events. Neuron. 2011; 69(2):359-72.

29. Lappalainen T, Greally JM. Associating cellular epigenetic models with human phenotypes. Nat Rev Genet. 2017;18(7):441.

30. Oken E, Baccarelli AA, Gold DR, Kleinman KP, Litonjua AA, De Meo D, et al. Cohort profile: project viva. Int J Epidemiol. 2014;44(1):37-48.

31. Wadhwa PD, Sandman CA, Porto M, Dunkel-Schetter C, Garite TJ. The association between prenatal stress and infant birth weight and gestational age at birth: a prospective investigation. American Journal of Obstetrics \& Gynecology. 1993;169(4):858-65.

32. Rini CK, Dunkel-Schetter C, Wadhwa PD, Sandman CA. Psychological adaptation and birth outcomes: the role of personal resources, stress, and sociocultural context in pregnancy. Health Psychol. 1999;18(4):333.

33. Cox JL, Holden JM, Sagovsky R. Detection of postnatal depression: development of the 10-item Edinburgh Postnatal Depression Scale. Br J Psychiatry. 1987;150(6):782-6. 
34. Murray D, Cox JL. Screening for depression during pregnancy with the Edinburgh Depression Scale (EDDS). Journal of Reproductive and Infant Psychology. 1990;8(2):99-107.

35. Evans J, Heron J, Francomb H, Oke S, Golding J. Cohort study of depressed mood during pregnancy and after childbirth. BMJ. 2001;323(7307):257-60.

36. Matthey S, Henshaw C, Elliott S, Barnett B. Variability in use of cut-off scores and formats on the Edinburgh Postnatal Depression Scale-implications for clinical and research practice. Archives of Women's Mental Health. 2006;9(6):309-15.

37. Triche TJ Jr, Weisenberger DJ, Van Den Berg D, Laird PW, Siegmund KD. Low-level processing of Illumina Infinium DNA methylation beadarrays. Nucleic Acids Res. 2013:41(7):e90

38. Teschendorff AE, Marabita F, Lechner M, Bartlett T, Tegner J, Gomez-Cabrero $\mathrm{D}$, et al. A beta-mixture quantile normalization method for correcting probe design bias in Illumina Infinium 450 k DNA methylation data. Bioinformatics. 2012;29(2):189-96

39. Y-a C, Lemire M, Choufani S, Butcher DT, Grafodatskaya D, Zanke BW, et al. Discovery of cross-reactive probes and polymorphic CpGs in the Illumina Infinium HumanMethylation450 microarray. Epigenetics. 2013;8(2):203-9.

40. Leek JT, Johnson WE, Parker HS, Jaffe AE, Storey JD. The sva package for removing batch effects and other unwanted variation in high-throughput experiments. Bioinformatics. 2012;28(6):882-3.

41. Du P, Zhang X, Huang C-C, Jafari N, Kibbe WA, Hou L, et al. Comparison of Beta-value and $\mathrm{M}$-value methods for quantifying methylation levels by microarray analysis. BMC Bioinformatics. 2010;11(1):587.

42. Kooijman MN, Kruithof $\mathrm{CJ}$, van Duijn CM, Duijts L, Franco OH, van IJzendoorn MH, et al. The Generation R Study: design and cohort update 2017. Eur J Epidemiol. 2016;31(12):1243-64.

43. Lehne B, Drong AW, Loh M, Zhang W, Scott WR, Tan S-T, et al. A coherent approach for analysis of the Illumina HumanMethylation450 BeadChip improves data quality and performance in epigenome-wide association studies. Genome Biol. 2015;16(1):37.

44. Team RC. R: a language and environment for statistical computing; 2013.

45. Derogatis $L R$, Melisaratos $N$. The brief symptom inventory: an introductory report. Psychol Med. 1983;13(3):595-605

46. De Beurs E. Brief Symptom Inventory, handleiding addendum. Leiden, The Netherlands: PITS BV. 2009

47. Guxens M, Sonnenschein-van der Voort AM, Tiemeier H, Hofman A, Sunyer $J$, de Jongste JC, et al. Parental psychological distress during pregnancy and wheezing in preschool children: the Generation R Study. J Allergy Clin Immunol. 2014;133(1):59-67 e12.

48. El Marroun H, Jaddoe WW, Hudziak JJ, Roza SJ, Steegers EA, Hofman A, et al. Maternal use of selective serotonin reuptake inhibitors, fetal growth, and risk of adverse birth outcomes. Arch Gen Psychiatry. 2012;69(7):706-14.

49. Aryee MJ, Jaffe AE, Corrada-Bravo H, Ladd-Acosta C, Feinberg AP, Hansen KD, et al. Minfi: a flexible and comprehensive Bioconductor package for the analysis of Infinium DNA methylation microarrays. Bioinformatics. 2014;30(10):1363-9.

50. Peters TJ, Buckley MJ, Statham AL, Pidsley R, Samaras K, Lord RV, et al. De novo identification of differentially methylated regions in the human genome. Epigenetics Chromatin. 2015;8(1):6.

51. Hannon E, Lunnon K, Schalkwyk L, Mill J. Interindividual methylomic variation across blood, cortex, and cerebellum: implications for epigenetic studies of neurological and neuropsychiatric phenotypes. Epigenetics. 2015; 10(11):1024-32.

Ready to submit your research? Choose BMC and benefit from:

- fast, convenient online submission

- thorough peer review by experienced researchers in your field

- rapid publication on acceptance

- support for research data, including large and complex data types

- gold Open Access which fosters wider collaboration and increased citations

- maximum visibility for your research: over $100 \mathrm{M}$ website views per year

At $\mathrm{BMC}$, research is always in progress.

Learn more biomedcentral.com/submissions 